EGU21-9388, updated on 18 Jan 2022

https://doi.org/10.5194/egusphere-egu21-9388

EGU General Assembly 2021

(c) Author(s) 2022. This work is distributed under

the Creative Commons Attribution 4.0 License.

\title{
Disentangling the mechanisms of wave-convection coupling using idealized simulations in a tropical channel: wave composites of the moist static energy budget
}

\author{
Hyunju Jung ${ }^{1}$, Peter Knippertz ${ }^{1}$, Corinna Hoose ${ }^{1}$, Yvonne Ruckstuhl ${ }^{2}$, Robert Redl ${ }^{2}$, and Tijana \\ Janjic $^{2}$ \\ ${ }^{1}$ Institute of Meteorology and Climate Research, Karlsruhe Institute of Technology, Karlsruhe, Germany \\ ${ }^{2}$ Institute of Meteorology, Ludwig Maximilian University of Munich, Munich, Germany
}

Recent studies found that the coupling of equatorial waves to convection is key to improving weather forecasts in the tropics on the synoptic to the subseasonal timescale but many models struggle to realistically represent this coupling. To study the underlying mechanisms of convectively coupled equatorial waves, we use aquaplanet simulations with the ICOsahedral Nonhydrostatic (ICON) model in a tropical channel configuration with a horizontal grid spacing of $13 \mathrm{~km}$ and with a prescribed zonally symmetric, latitudinally varying sea surface temperature. We compare simulations with parameterized and explicit deep/shallow convection. Using wave identification tools that are based on Fourier filtering in time and space and on projections of dynamical fields on theoretical wave patterns, we observe a predominance of equator-symmetric equatorial waves such as Kelvin waves and slow large-scale variability resembling the MaddenJulian Oscillation.

To diagnose interactions between the equatorial waves and convection, we use a moist static energy (MSE) framework. A budget analysis for column integrated MSE shows that spatial anomalies of the net shortwave and longwave radiation and the surface enthalpy flux increase the spatial variance of the column MSE, while advection dampens variability. For wave-convection coupling we employ a wave composite technique for the terms of the MSE budget. Results from this analysis will be presented at the conference. The same filtering tools and diagnostics are applied to a realistic ICON simulation with a $2.5 \mathrm{~km}$ horizontal grid spacing from the DYnamics of the Atmospheric general circulation Modeled On Non-hydrostatic Domains (DYAMOND) project. 